\title{
Effect of Natural Ageing and Seed Priming on Field Emergence of Indian Mustard [Brassica juncea L. (Czern \& coss)]
}

\author{
Pradeep Singh*, R.C. Punia, V.S. Mor and Sunil Kumar \\ Department of Seed Science and Technology, CCS Haryana Agricultural University, \\ Hisar-125004, Haryana, India \\ *Corresponding author
}

\begin{tabular}{|c|}
\hline Keywords \\
\hline $\begin{array}{l}\text { Indian mustard, } \\
\text { Seed lot, Natural } \\
\text { ageing and seed } \\
\text { priming }\end{array}$ \\
\hline Article Info \\
\hline $\begin{array}{l}\text { Accepted: } \\
\text { 26 February } 2018 \\
\text { Available Online } \\
10 \text { March } 2018\end{array}$ \\
\hline
\end{tabular}

A B S T R A C T

A field experiment was conducted at CCS Haryana Agricultural University, Hisar to study the effect of natural ageing and seed priming on field parameters of Indian mustard. Three variety of Indian mustard viz., RH-30, RH-9304 and RH-0406 with three seed lot were evaluated for vigour and viability parameters under field conditions. The results revealed that seed lot ' $\mathrm{L}_{1}$ ' was found maximum seedling emergence index and seedling establishment followed by seed lot ' $\mathrm{L}_{2}$ ' and minimum seedling emergence and seedling establishment was found in the seed lot ' $\mathrm{L}_{3}$ ' during the year 2015-16 and 2016-17. Among the variety, 'RH-30' was found maximum seedling emergence and seedling establishment followed by variety 'RH-9304' and minimum seedling emergence index and seedling establishment was recorded in the variety ' $\mathrm{RH}-0406$ ' during both the year of study. In case of mean emergence time maximum mean emergence time was found in the seed lot ' $\mathrm{L}_{3}$ ' and minimum mean emergence time was found in the seed lot ' $\mathrm{L}_{1}$ '. Variety RH-0406 was found highest mean emergence time followed by 'RH-9304' and lowest mean emergence time was found in the variety ' $\mathrm{RH}-30$ '. All seed priming treatment enhanced the seed quality significantly in case of all physiological parameters in naturally aged seed lots of different varieties of Indian mustard. Among different priming treatments, hydration with $\mathrm{GA}_{3}$ (@50 ppm) was reported superior for enhancing the seedling emergence and seedling establishment of all the seed lots of different varieties of Indian mustard. Maximum enhancement was found in the variety $\mathrm{RH}-30$ followed by $\mathrm{RH}-9304$ and minimum improvement was recorded in the variety 'RH-0406' during both the year. It can be concluded that seed treatment with $\mathrm{GA}_{3}$ could be used for enhancing the field emergence of Indian mustard.

\section{Introduction}

Indian mustard [Brassica juncea L. (Czern \& coss)] a member of Brassicaceae family and an important oil seed crop of the world. Population of India is increasing rapidly and consequently edible oil demand is also going up day by day. In India Rapeseed and mustard is grown in an area of $5.76 \mathrm{M}$ ha with production and productivity of $6.8 \mathrm{MT}$ and $1184 \mathrm{Kg} / \mathrm{ha}$, respectively (Anonymous, 2016).

Most popular varieties grown in Indian subcontinent are Brassica juncea, Brassica campestris and Brassica napus L. Brassica juncea is a highly variable species which has 
been cultivated for centuries as an oil plant. The crop is globally gaining importance relative to other crops due to higher oil content in the seed and higher yield potential, with higher return at low cost of production, low moisture requirement, wider adaptability for various farming conditions. Despite, these facts the area, production and yield of rapeseed mustard in India is fluctuating due to various problems associated with this crop.

Seed being a living entity, deterioration in its quality occurs with the advancement in ageing which is natural, inevitable, irreversible and continuous process. Seed ageing is recognized by some parameters like delay in germination and emergence, slow growth and increasing of susceptibility to environmental stresses.

Biotic and abiotic factors affect seed vigour and quality till they are on mother plants. Genetic structure, environmental condition during seed development and condition of stresses mainly effect on seed vigour. Using vigorous seeds at planting may increase crop yield in two ways: freshly, higher percentage of seedling emergence than aged or weaken seeds that gives optimum density even under stressful conditions and secondly, high growth and emergence rate in comparison to seedlings produced from aged seeds (ghasemi-golezani et al., 2010).

Seed priming (osmo conditioning) has been successfully demonstrated to improve seed germination and seedling establishment for many field crops, such as wheat, sugar beet, maize, soybean, and sunflower (Singh 1995; Khajeh-Hosseini et al., 2003; Sadeghian and Yavari, 2004).

The beneficial effects of priming have also been demonstrated on germination and seed emergence, as well as seedling establishment of many crops, particularly under salt stress, as reported by Patane et al., (2009), who indicated that osmo conditioning of sweet sorghum seeds mitigated the harmful effects caused by salt and thermal stress during germination and radicle growth. Furthermore, in artificially aged seeds, the literature indicates beneficial effects of osmo conditioning associated with repair and buildup of nucleic acids, increased synthesis of proteins and repair of membranes (Lanteri et al., 1998; McDonald 2000). Priming also enhances the activities of anti-oxidation in treated seeds (Hsu et al., 2003). Therefore, the improved seed quality promoted by seed priming has been attributable principally to reduced lipid peroxidation, resulting from enhanced antioxidative activities (Chiu et al., 2006).

\section{Materials and Methods}

The seeds of each seed lot $\mathrm{L}_{1}$ (Fresh seed), $\mathrm{L}_{1}$ (one year old seed) and $\mathrm{L}_{3}$ (Two year old seed) of three varieties (RH-30, RH-9304 and $\mathrm{RH}-0406)$ were stored under ambient conditions in the laboratory of Seed Science and Technology. Five priming treatments were used, namely carbendazim $(2 \mathrm{~g} / \mathrm{kg}), \mathrm{GA}_{3}(50$ ppm), $\mathrm{KNO}_{3}(0.5 \%), \mathrm{KH}_{2} \mathrm{PO}_{4}(0.5 \%)$ and an absolute control (without priming) was also maintained.

All 3 seed lots of each variety of experiment were laid out in factorial Randomized Block Design (RBD) for field studies with three replications during 2015-16 and 2016-17. In each replication 100 seeds were planted during 2015-16 and 2016-17. The observations were recorded at every six months interval up to two year on the above seed lots on the following parameters. Statistical analysis of data collected during the study was done by applying the technique of analysis of variance (ANOVA) as suggested by Gomez and Gomez (1984) and Panse and Sukhatme (1961). All the statistical analysis was carried out by using OPSTAT statistical software. 


\section{Results and Discussion}

\section{Effect of naturally aged seed lots on field performance of Indian mustard}

\section{Seedling emergence index}

Significant differences were observed for the effect of naturally aged Indian mustard seed lot on seedling emergence index (Table 1) during the year 2015-16 and 2016-17. It ranged from 4.38-8.42, 3.47-7.65, 2.86-6.17 and 2.24-5.70 during both of the years of study. Significantly, maximum seedling emergence index $(8.42,7.65,6.17$ and 5.70) was observed for the seed lot ' $\mathrm{L}_{1}$ ' during both of the years of study, whereas, minimum seedling emergence index $(4.38,3.47,2.86$ and 2.24) was recorded for the seed lot ' $\mathrm{L}_{3}$ ' during the year 2015-16 and 2016-17, which was found significantly lowest among all seed lots. The effect of different Indian mustard varieties, significant results were observed for this trait under study. However, maximum seedling emergence index $(8.36,7.59,6.59$ and 5.80) recorded for the variety RH-30 and minimum seedling emergence index (4.95, $4.28,3.04$ and 2.48) was observed in the variety RH-0406 during both of the year of study.

The interaction between different seed lots and varieties, seedling emergence index ranged from 2.80-10.14, 2.03-9.52, 1.53-8.29 and 1.02-7.74 during the year 2015-16 and 201617 , respectively.

The treatment combination ' $\mathrm{L}_{1} \times \mathrm{RH}-30$ ' recorded highest seedling emergence index $(10.14,9.52,8.29$ and 7.74), whereas, minimum seedling emergence index (2.80, 2.03, 1.53 and 1.02) was observed with the interaction of ' $\mathrm{L}_{3} \times \mathrm{RH}-0406$ ' during the year 2015-16 and 2016-17, respectively. The present investigation confirms the finding of Khajeh-Hosseini (2010) in rapeseed and mustard; in mungbean by Verma et al., (2003); and in wheat by Soltani et al., (2008 and 2009).

\section{Mean emergence time (MET)}

Significant differences was observed for the effect of naturally aged Indian mustard seed lot on mean emergence time during the year 2015-16 and 2016-17, respectively (Table 2). Mean emergence time ranged from 7.94-9.48, 8.27-9.90, 8.50, 10.37 and 8.68-10.83 during both of the years. Significantly minimum mean emergence index $(7.94,8.27,8.50$ and 8.68) was recorded in the seed lot ' $\mathrm{L}_{1}$ ' while, maximum mean emergence time $(9.48,9.90$, 10.37 and 10.83) was observed for the seed lot ' $\mathrm{L}_{3}$ ' during the year 2015-16 and 2016-17, which was found significantly highest among all the seed lots.

The effect of different seed lots and varieties, significant results obtained during both the year of study for this trait. However, maximum mean emergence time $(9.95,10.37$, 10.86 and 11.34) for the variety $\mathrm{RH}-0406$ followed by $(9.03,9.13,9.84$ and 10.01) for the variety RH-9304 and minimum mean emergence time $(6.95,7.32,7.66$ and 8.09) for the variety RH-30 during the year 2015-16 and 2016-17, respectively.

The interaction between different seed lots and varieties, mean emergence time ranged from 6.37-10.97, 6.64-11.35, 6.81-11.83 and 7.2912.36 was recorded during both of the years of study. The treatment combination ' $\mathrm{L}_{1} \times \mathrm{RH}$ 30 ' was recorded minimum mean emergence time $(6.37,6.64,6.81$ and 7.29), whereas, maximum mean emergence time (10.97, $11.35,11.83$ and 12.36) was observed with the interaction ' $\mathrm{L}_{3} \times \mathrm{RH}-0406$ ' during the year 2015-16 and 2016-17, respectively. Similar findings were observed in fenugreek by Singh et al., (2015) and Rai et al., (2017) in Indian mustard. 
Table.1 Effect of naturally aged seed lots on seedling emergence index of Indian mustard during 2015-16 and 2016-17

\begin{tabular}{|c|c|c|c|c|c|c|c|c|c|c|c|c|c|c|c|c|}
\hline \multicolumn{17}{|c|}{ Seed lot } \\
\hline Months & \multicolumn{4}{|c|}{0 month } & \multicolumn{4}{|c|}{ After 6 months } & \multicolumn{4}{|c|}{ After 12 months } & \multicolumn{4}{|c|}{ After 18 months } \\
\hline VARIETY & $\mathbf{L}_{1}$ & $\mathbf{L}_{2}$ & $\mathbf{L}_{3}$ & MEAN & $\mathbf{L}_{1}$ & $\mathbf{L}_{2}$ & $\mathbf{L}_{3}$ & MEAN & $\mathbf{L}_{1}$ & $\mathbf{L}_{2}$ & $\mathbf{L}_{3}$ & MEAN & $\mathbf{L}_{1}$ & $\mathbf{L}_{2}$ & $\mathbf{L}_{3}$ & MEAN \\
\hline RH-30 & 10.14 & 8.27 & 6.68 & 8.36 & 9.52 & 7.78 & 5.47 & 7.59 & 8.29 & 6.62 & 4.87 & 6.59 & 7.74 & 5.43 & 4.22 & 5.80 \\
\hline RH-9304 & 7.87 & 5.42 & 3.67 & 5.65 & 6.92 & 5.01 & 2.92 & 4.95 & 5.43 & 3.61 & 2.18 & 3.74 & 5.03 & 2.88 & 1.48 & 3.13 \\
\hline RH-0406 & 7.27 & 4.77 & 2.80 & 4.95 & 6.52 & 4.30 & 2.03 & 4.28 & 4.80 & 2.80 & 1.53 & 3.04 & 4.33 & 2.08 & 1.02 & 2.48 \\
\hline MEAN & 8.42 & 6.15 & 4.38 & & 7.65 & 5.70 & 3.47 & & 6.17 & 4.34 & 2.86 & & 5.70 & 3.46 & 2.24 & \\
\hline & \multicolumn{6}{|c|}{ C.D. } & \multicolumn{5}{|c|}{ SE(d) } & \multicolumn{5}{|c|}{ SE(m) } \\
\hline $\mathbf{L}$ & \multicolumn{6}{|c|}{0.028} & \multicolumn{5}{|c|}{0.014} & \multicolumn{5}{|c|}{0.01} \\
\hline MxL & \multicolumn{6}{|c|}{0.056} & \multicolumn{5}{|c|}{0.028} & \multicolumn{5}{|c|}{0.02} \\
\hline $\mathbf{V}$ & \multicolumn{6}{|c|}{0.028} & \multicolumn{5}{|c|}{0.014} & \multicolumn{5}{|c|}{0.01} \\
\hline $\mathbf{M x V}$ & \multicolumn{6}{|c|}{0.056} & \multicolumn{5}{|c|}{0.028} & \multicolumn{5}{|c|}{0.02} \\
\hline $\mathbf{L x V}$ & \multicolumn{6}{|c|}{0.048} & \multicolumn{5}{|c|}{0.024} & \multicolumn{5}{|c|}{0.017} \\
\hline MxLxV & \multicolumn{6}{|c|}{0.097} & \multicolumn{5}{|c|}{0.048} & \multicolumn{5}{|c|}{0.034} \\
\hline
\end{tabular}

Table.2 Effect of naturally aged seed lots on mean emergence time of Indian mustard during 2015-16 and 2016-17

\begin{tabular}{|c|c|c|c|c|c|c|c|c|c|c|c|c|c|c|c|c|}
\hline \multicolumn{17}{|c|}{ Seed lot } \\
\hline Months & \multicolumn{4}{|c|}{0 month } & \multicolumn{4}{|c|}{ After 6 months } & \multicolumn{4}{|c|}{ After 12 months } & \multicolumn{4}{|c|}{ After 18 months } \\
\hline VARIETY & $\mathbf{L}_{1}$ & $\mathbf{L}_{2}$ & $\mathbf{L}_{3}$ & MEAN & $\mathbf{L}_{1}$ & $\mathbf{L}_{2}$ & $\mathbf{L}_{3}$ & MEAN & $\mathbf{L}_{1}$ & $\mathbf{L}_{2}$ & $\mathbf{L}_{3}$ & MEAN & $\mathbf{L}_{1}$ & $\mathbf{L}_{2}$ & $\mathbf{L}_{3}$ & MEAN \\
\hline RH-30 & 6.37 & 6.84 & 7.63 & 6.95 & 6.64 & 7.27 & 8.06 & 7.32 & 6.81 & 7.65 & 8.51 & 7.66 & 7.29 & 8.09 & 8.88 & 8.09 \\
\hline RH-9304 & 8.33 & 8.91 & 9.84 & 9.03 & 8.67 & 8.46 & 10.28 & 9.13 & 8.91 & 9.85 & 10.77 & 9.84 & 8.48 & 10.29 & 11.25 & 10.01 \\
\hline RH-0406 & 9.11 & 9.77 & 10.97 & 9.95 & 9.50 & 10.26 & 11.35 & 10.37 & 9.78 & 10.97 & 11.83 & 10.86 & 10.28 & 11.38 & 12.36 & 11.34 \\
\hline MEAN & 7.94 & 8.51 & 9.48 & & 8.27 & 8.66 & 9.90 & & 8.50 & 9.49 & 10.37 & & 8.68 & 9.92 & 10.83 & \\
\hline & \multicolumn{6}{|c|}{ C.D. } & \multicolumn{5}{|c|}{ SE(d) } & \multicolumn{5}{|c|}{ SE(m) } \\
\hline $\mathbf{L}$ & \multicolumn{6}{|c|}{0.038} & \multicolumn{5}{|c|}{0.019} & \multicolumn{5}{|c|}{0.014} \\
\hline MxL & \multicolumn{6}{|c|}{0.077} & \multicolumn{5}{|c|}{0.038} & \multicolumn{5}{|c|}{0.027} \\
\hline $\mathbf{V}$ & \multicolumn{6}{|c|}{0.038} & \multicolumn{5}{|c|}{0.019} & \multicolumn{5}{|c|}{0.014} \\
\hline $\mathbf{M x V}$ & \multicolumn{6}{|c|}{0.077} & \multicolumn{5}{|c|}{0.038} & \multicolumn{5}{|c|}{0.027} \\
\hline $\mathbf{L x V}$ & \multicolumn{6}{|c|}{0.066} & \multicolumn{5}{|c|}{0.033} & \multicolumn{5}{|c|}{0.024} \\
\hline MxLxV & \multicolumn{6}{|c|}{0.133} & \multicolumn{5}{|c|}{0.067} & \multicolumn{5}{|c|}{0.047} \\
\hline
\end{tabular}


Table.3 Effect of naturally aged seed lots on seedling establishment (\%) of Indian mustard during 2015-16 and 2016-17

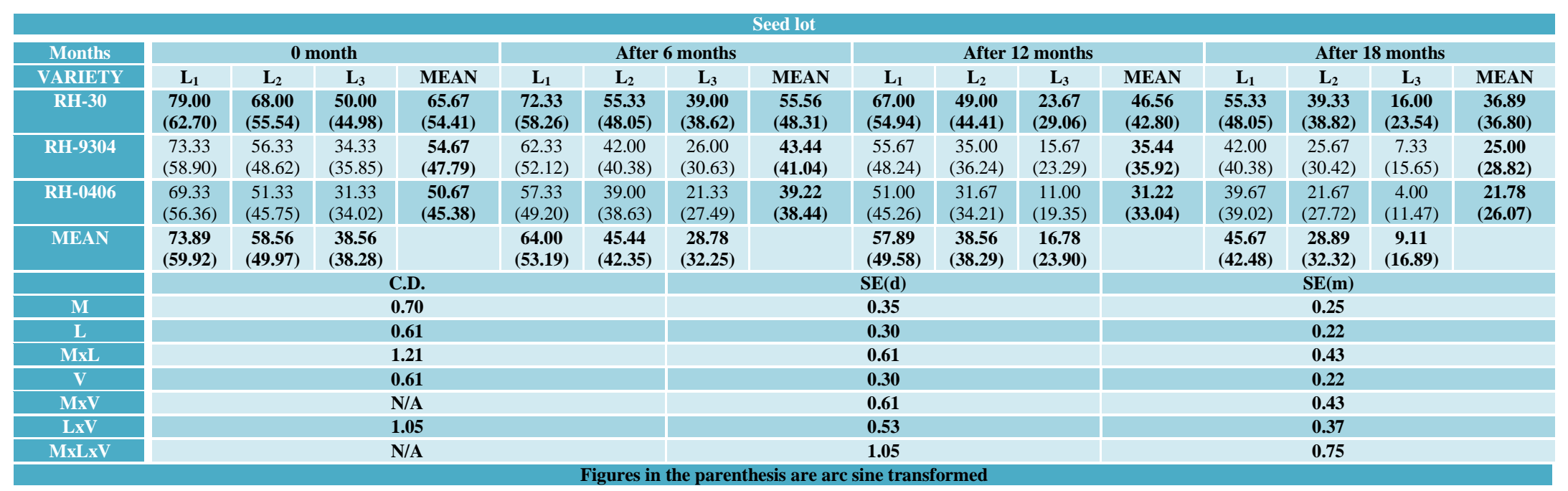

Table.4 Effect of seed priming treatments on seedling emergence index of naturally aged seed lots of Indian mustard during 2015-16 and 2016-17

\begin{tabular}{|c|c|c|c|c|c|c|c|c|c|c|c|c|c|c|c|c|c|c|c|c|c|c|c|c|}
\hline \multirow{3}{*}{$\begin{array}{l}\text { Year } \\
\text { Variety } \\
\text { Lots }\end{array}$} & \multicolumn{12}{|c|}{ 2015-16 } & \multicolumn{12}{|c|}{ 2016-17 } \\
\hline & \multicolumn{4}{|c|}{ RH-30 } & \multicolumn{4}{|c|}{ RH-9304 } & \multicolumn{4}{|c|}{ RH-0406 } & \multicolumn{4}{|c|}{ RH-30 } & \multicolumn{4}{|c|}{ RH-9304 } & \multicolumn{4}{|c|}{ RH-0406 } \\
\hline & $\mathrm{L}_{1}$ & $\mathrm{~L}_{2}$ & $\mathrm{~L}_{3}$ & Mean & $\mathrm{L}_{1}$ & $\mathrm{~L}_{2}$ & $\mathrm{~L}_{3}$ & Mean & $\mathrm{L}_{1}$ & $\mathrm{~L}_{2}$ & $\mathrm{~L}_{3}$ & Mean & $\mathrm{L}_{1}$ & $\mathrm{~L}_{2}$ & $\mathrm{~L}_{3}$ & Mean & $\mathrm{L}_{1}$ & $\mathrm{~L}_{2}$ & $\mathrm{~L}_{3}$ & Mean & $\mathrm{L}_{1}$ & $\mathrm{~L}_{2}$ & $\mathrm{~L}_{3}$ & Mean \\
\hline $\mathrm{T}_{0}$ & 10.14 & 8.27 & 6.68 & 8.36 & 7.87 & 5.42 & 3.67 & 5.65 & 7.27 & 4.77 & 2.80 & 4.95 & 7.74 & 5.43 & 4.22 & 5.80 & 5.03 & 2.88 & 1.48 & 3.13 & 4.33 & 2.08 & 1.02 & 2.48 \\
\hline$T_{1}$ & 10.74 & 9.16 & 7.84 & 9.24 & 8.41 & 6.23 & 4.68 & 6.44 & 7.95 & 5.64 & 3.82 & 5.80 & 8.66 & 6.24 & 4.76 & 6.55 & 5.84 & 3.60 & 1.94 & 3.79 & 5.27 & 2.94 & 1.61 & 3.28 \\
\hline$T_{2}$ & 11.11 & 9.80 & 8.62 & 9.84 & 8.78 & 6.84 & 5.35 & 6.99 & 8.08 & 6.00 & 4.33 & 6.14 & 9.32 & 6.74 & 5.03 & 7.03 & 6.44 & 4.07 & 2.19 & 4.23 & 5.53 & 3.14 & 1.71 & 3.46 \\
\hline$T_{3}$ & 10.51 & 9.13 & 7.74 & 9.12 & 8.18 & 6.21 & 4.60 & 6.33 & 7.58 & 5.46 & 3.68 & 5.57 & 8.63 & 6.19 & 4.68 & 6.50 & 5.81 & 3.53 & 1.88 & 3.74 & 4.94 & 2.69 & 1.41 & 3.02 \\
\hline $\mathrm{T}_{4}$ & 10.49 & 9.10 & 7.65 & 9.08 & 8.14 & 6.13 & 4.53 & 6.26 & 7.53 & 5.39 & 3.54 & 5.49 & 8.58 & 6.12 & 4.64 & 6.45 & 5.74 & 3.47 & 1.82 & 3.68 & 4.92 & 2.67 & 1.35 & 2.98 \\
\hline $\mathbf{T}_{5}$ & 10.96 & 9.38 & 8.11 & 9.48 & 8.63 & 6.40 & 4.88 & 6.63 & 7.74 & 5.50 & 3.71 & 5.65 & 8.92 & 6.44 & 4.89 & 6.75 & 6.05 & 3.79 & 2.03 & 3.96 & 5.04 & 2.75 & 1.49 & 3.10 \\
\hline \multirow[t]{2}{*}{ Mean } & 10.66 & 9.14 & 7.77 & & 8.33 & 6.21 & 4.62 & & 7.69 & 5.46 & 3.64 & & 8.64 & 6.19 & 4.71 & & 5.82 & 3.56 & 1.89 & & 5.01 & 2.71 & 1.43 & \\
\hline & \multicolumn{4}{|c|}{ C.D. } & \multicolumn{4}{|c|}{ SE(d) } & \multicolumn{4}{|c|}{ SE $(m)$} & \multicolumn{4}{|c|}{ C.D. } & \multicolumn{4}{|c|}{$\mathrm{SE}(\mathrm{d})$} & \multicolumn{4}{|c|}{$\mathrm{SE}(\mathrm{m})$} \\
\hline $\mathbf{v}$ & \multicolumn{4}{|c|}{0.017} & \multicolumn{4}{|c|}{0.008} & \multicolumn{4}{|c|}{0.006} & \multicolumn{4}{|c|}{0.022} & \multicolumn{4}{|c|}{0.011} & \multicolumn{4}{|c|}{0.008} \\
\hline $\mathbf{L}$ & \multirow{2}{*}{\multicolumn{4}{|c|}{0.017}} & \multirow{2}{*}{\multicolumn{4}{|c|}{0.008}} & \multicolumn{4}{|c|}{0.006} & \multicolumn{4}{|c|}{0.022} & \multicolumn{4}{|c|}{0.011} & \multicolumn{4}{|c|}{0.008} \\
\hline $\mathrm{VxL}$ & & & & & & & & & & & 01 & & & & 38 & & & & & & & & & \\
\hline $\mathrm{T}$ & & & & & & & 12 & & & & 008 & & & & 31 & & & & & & & & & \\
\hline $\mathrm{V} \times \mathrm{C}$ & & & & & & & 02 & & & & 14 & & & & 54 & & & & & & & & & \\
\hline $\mathbf{L x} \mathrm{T}$ & & & & & & & 02 & & & & 14 & & & & 54 & & & & & & & & & \\
\hline $\mathrm{V} \times \mathrm{L} \times \mathrm{T}$ & & & & & & & 35 & & & & 25 & & & & A & & & & & & & & & \\
\hline
\end{tabular}


Table.5 Effect of seed priming treatments on mean emergence time of naturally aged seed lots of Indian mustard during 2015-16 and 2016-17

\begin{tabular}{|c|c|c|c|c|c|c|c|c|c|c|c|c|c|c|c|c|c|c|c|c|c|c|c|c|}
\hline \multirow{3}{*}{$\begin{array}{l}\text { Year } \\
\text { Variety } \\
\text { Lots }\end{array}$} & \multicolumn{12}{|c|}{ 2015-16 } & \multicolumn{12}{|c|}{ 2016-17 } \\
\hline & \multicolumn{4}{|c|}{ RH-30 } & \multicolumn{4}{|c|}{ RH-9304 } & \multicolumn{4}{|c|}{ RH-0406 } & \multicolumn{4}{|c|}{ RH-30 } & \multicolumn{4}{|c|}{ RH-9304 } & \multicolumn{4}{|c|}{ RH-0406 } \\
\hline & $\mathrm{L}_{1}$ & $\mathrm{~L}_{2}$ & $\mathrm{~L}_{3}$ & Mean & $\mathrm{L}_{1}$ & $\mathrm{~L}_{2}$ & $\mathrm{~L}_{3}$ & Mean & $\mathrm{L}_{1}$ & $\mathrm{~L}_{2}$ & $\mathrm{~L}_{3}$ & Mean & $\mathrm{L}_{1}$ & $\mathrm{~L}_{2}$ & $\mathrm{~L}_{3}$ & Mean & $\mathrm{L}_{1}$ & $\mathrm{~L}_{2}$ & $\mathrm{~L}_{3}$ & Mean & $\mathrm{L}_{1}$ & $\mathrm{~L}_{2}$ & $\mathrm{~L}_{3}$ & Mean \\
\hline$T_{0}$ & 6.37 & 6.84 & 7.63 & 6.95 & 8.33 & 8.91 & 9.84 & 9.03 & 9.11 & 9.77 & 10.97 & 9.95 & 7.29 & 8.09 & 8.88 & 8.09 & 8.48 & 10.29 & 11.25 & 10.01 & 10.28 & 11.38 & 12.36 & 11.34 \\
\hline$T_{1}$ & 5.67 & 5.92 & 6.53 & 6.04 & 7.76 & 8.05 & 8.86 & 8.22 & 8.43 & 8.73 & 9.95 & 9.04 & 6.43 & 7.39 & 8.25 & 7.36 & 7.67 & 9.65 & 10.67 & 9.33 & 9.44 & 10.69 & 11.75 & 10.63 \\
\hline$T_{2}$ & 5.36 & 5.49 & 5.81 & 5.56 & 7.44 & 7.62 & 8.10 & 7.72 & 8.30 & 8.64 & 9.44 & 8.79 & 6.03 & 6.94 & 7.87 & 6.95 & 7.30 & 9.28 & 10.32 & 8.97 & 9.24 & 10.49 & 11.52 & 10.42 \\
\hline$T_{3}$ & 5.90 & 5.95 & 6.60 & 6.15 & 7.91 & 8.07 & 9.04 & 8.34 & 8.73 & 9.04 & 10.26 & 9.34 & 6.60 & 7.55 & 8.39 & 7.51 & 7.87 & 9.80 & 10.86 & 9.51 & 9.74 & 10.97 & 11.97 & 10.89 \\
\hline$T_{4}$ & 5.96 & 6.03 & 6.65 & 6.22 & 7.95 & 8.15 & 9.09 & 8.40 & 8.80 & 9.08 & 10.33 & 9.40 & 6.68 & 7.58 & 8.44 & 7.57 & 7.94 & 9.88 & 10.94 & 9.59 & 9.79 & 11.04 & 12.05 & 10.96 \\
\hline$T_{5}$ & 5.55 & 5.67 & 6.31 & 5.85 & 7.59 & 7.69 & 8.59 & 7.96 & 8.60 & 8.96 & 10.06 & 9.21 & 6.31 & 7.25 & 8.12 & 7.23 & 7.59 & 9.58 & 10.54 & 9.24 & 9.57 & 10.77 & 11.85 & 10.73 \\
\hline \multirow[t]{2}{*}{ Mean } & 5.80 & 5.99 & 6.59 & & 7.83 & 8.08 & 8.92 & & 8.66 & 9.04 & 10.17 & & 6.55 & 7.47 & 8.33 & & 7.81 & 9.75 & 10.76 & & 9.68 & 10.89 & 11.92 & \\
\hline & \multirow{2}{*}{\multicolumn{4}{|c|}{ C.D. }} & \multicolumn{4}{|c|}{$\mathrm{SE}(\mathbf{d})$} & \multicolumn{4}{|c|}{ SE(m) } & \multicolumn{4}{|c|}{ C.D. } & \multicolumn{4}{|c|}{ SE(d) } & \multicolumn{4}{|c|}{ SE(m) } \\
\hline $\mathbf{v}$ & & & & & & \multicolumn{2}{|c|}{$\begin{array}{l}0.017 \\
0.017\end{array}$} & & \multicolumn{4}{|c|}{$\begin{array}{l}0.012 \\
0.012\end{array}$} & & & & & & 0.0 & & & & & & \\
\hline $\mathrm{VxL}$ & \multicolumn{4}{|c|}{0.058} & \multicolumn{4}{|c|}{0.029} & \multicolumn{4}{|c|}{0.021} & \multicolumn{4}{|c|}{0.047} & \multicolumn{4}{|c|}{0.024} & \multicolumn{4}{|c|}{0.017} \\
\hline $\mathrm{T}$ & & & & & & & & & & & & & & & & & & 0.0 & & & & & & \\
\hline $\mathrm{VxC}$ & & & & & & & & & & & & & & & & & & 0.0 & & & & & & \\
\hline $\mathrm{L} \times \mathrm{T}$ & & & & & & & & & & & 29 & & & & & & & 0.0 & & & & & & \\
\hline VXLXT & & & & & & & & & & & 05 & & & & & & & 0.0 & & & & & & \\
\hline
\end{tabular}

Table.6 Effect of seed priming treatments on seedling establishment of naturally aged seed lots of Indian mustard during 2015-16 and 2016-17

\begin{tabular}{|c|c|c|c|c|c|c|c|c|c|c|c|c|c|c|c|c|c|c|c|c|c|c|c|c|}
\hline \multirow{3}{*}{\begin{tabular}{|l|} 
Year \\
Variety \\
Lots \\
\end{tabular}} & \multicolumn{12}{|c|}{$2015-16$} & \multicolumn{12}{|c|}{$2016-17$} \\
\hline & \multicolumn{4}{|c|}{ RH-30 } & \multicolumn{4}{|c|}{ RH-9304 } & \multicolumn{4}{|c|}{ RH-0406 } & \multicolumn{4}{|c|}{ RH-30 } & \multicolumn{4}{|c|}{ RH-9304 } & \multicolumn{4}{|c|}{ RH-0406 } \\
\hline & $\mathrm{L}_{1}$ & $\mathbf{L}_{2}$ & $\mathbf{L}_{3}$ & Mean & $\mathbf{L}_{1}$ & $\mathbf{L}_{2}$ & $\mathbf{L}_{3}$ & Mean & $\mathbf{L}_{1}$ & $\mathbf{L}_{2}$ & $\mathbf{L}_{3}$ & Mean & $\mathrm{L}_{1}$ & $\mathbf{L}_{2}$ & $\mathbf{L}_{3}$ & Mean & $\mathrm{L}_{1}$ & $\mathbf{L}_{2}$ & $\mathbf{L}_{3}$ & Mean & $\mathrm{L}_{1}$ & $\mathbf{L}_{2}$ & $\mathbf{L}_{3}$ & Mean \\
\hline$T_{0}$ & $\begin{array}{c}79.00 \\
(62.70)\end{array}$ & $\begin{array}{c}68.00 \\
(55.54)\end{array}$ & $\begin{array}{c}50.00 \\
(44.98)\end{array}$ & $\begin{array}{c}65.67 \\
(54.41)\end{array}$ & $\begin{array}{r}73.33 \\
(58.90)\end{array}$ & $\begin{array}{c}56.33 \\
(48.62)\end{array}$ & $\begin{array}{c}34.33 \\
(35.85) \\
\end{array}$ & $\begin{array}{c}54.67 \\
(47.79)\end{array}$ & $\begin{array}{r}69.33 \\
(56.36)\end{array}$ & $\begin{array}{c}51.33 \\
(45.75)\end{array}$ & $\begin{array}{c}31.33 \\
(34.02)\end{array}$ & $\begin{array}{c}50.67 \\
(45.38)\end{array}$ & $\begin{array}{c}55.00 \\
(47.85)\end{array}$ & $\begin{array}{c}39.00 \\
(38.63)\end{array}$ & $\begin{array}{c}16.00 \\
(23.54)\end{array}$ & $\begin{array}{c}36.67 \\
(36.67)\end{array}$ & $\begin{array}{c}42.00 \\
(40.38)\end{array}$ & $\begin{array}{c}25.67 \\
(30.42)\end{array}$ & $\begin{array}{c}7.00 \\
(15.31)\end{array}$ & $\begin{array}{c}24.89 \\
(28.70)\end{array}$ & $\begin{array}{c}39.33 \\
(38.82) \\
\end{array}$ & $\begin{array}{c}21.67 \\
(27.72)\end{array}$ & $\begin{array}{c}3.33 \\
(10.40)\end{array}$ & $\begin{array}{r}21.44 \\
(25.65)\end{array}$ \\
\hline$T_{1}$ & $\begin{array}{c}79.33 \\
(62.94)\end{array}$ & $\begin{array}{c}71.00 \\
(57.40)\end{array}$ & $\begin{array}{c}56.00 \\
(48.43)\end{array}$ & $\begin{array}{c}68.78 \\
(56.26)\end{array}$ & $\begin{array}{c}73.33 \\
(58.92)\end{array}$ & $\begin{array}{c}59.00 \\
(50.17)\end{array}$ & $\begin{array}{c}39.33 \\
(38.82)\end{array}$ & $\begin{array}{c}57.22 \\
(49.30)\end{array}$ & $\begin{array}{c}69.67 \\
(56.78)\end{array}$ & $\begin{array}{c}54.00 \\
(47.28)\end{array}$ & $\begin{array}{c}36.67 \\
(37.25)\end{array}$ & $\begin{array}{c}53.44 \\
(47.03)\end{array}$ & $\begin{array}{c}60.33 \\
(50.95)\end{array}$ & $\begin{array}{c}42.00 \\
(40.38)\end{array}$ & $\begin{array}{c}18.00 \\
(25.07)\end{array}$ & $\begin{array}{l}40.11 \\
(38.80)\end{array}$ & $\begin{array}{r}46.00 \\
(42.69)\end{array}$ & $\begin{array}{c}28.33 \\
(32.13)\end{array}$ & $\begin{array}{c}8.67 \\
(17.11)\end{array}$ & $\begin{array}{c}27.67 \\
(30.64)\end{array}$ & $\begin{array}{c}43.67 \\
(41.34)\end{array}$ & $\begin{array}{c}24.33 \\
\text { (29.53) }\end{array}$ & $\begin{array}{c}5.00 \\
(12.87)\end{array}$ & $\begin{array}{r}24.33 \\
(27.92)\end{array}$ \\
\hline $\mathbf{T}_{2}$ & $\begin{array}{c}80.33 \\
(63.65)\end{array}$ & $\begin{array}{c}73.00 \\
(58.68)\end{array}$ & $\begin{array}{c}60.00 \\
(50.75)\end{array}$ & $\begin{array}{c}71.11 \\
(57.69)\end{array}$ & $\begin{array}{c}74.33 \\
(59.54)\end{array}$ & $\begin{array}{c}60.33 \\
(50.95)\end{array}$ & $\begin{array}{c}42.00 \\
(40.38)\end{array}$ & $\begin{array}{c}58.89 \\
(50.29)\end{array}$ & $\begin{array}{c}70.00 \\
(56.77)\end{array}$ & $\begin{array}{c}54.67 \\
(47.66)\end{array}$ & $\begin{array}{c}38.67 \\
(38.43)\end{array}$ & $\begin{array}{c}54.44 \\
(47.62)\end{array}$ & $\begin{array}{c}64.33 \\
(53.32)\end{array}$ & $\begin{array}{l}43.67 \\
(41.35)\end{array}$ & $\begin{array}{c}19.67 \\
(26.26)\end{array}$ & $\begin{array}{c}42.56 \\
(40.31)\end{array}$ & $\begin{array}{c}49.00 \\
(44.41)\end{array}$ & $\begin{array}{c}29.33 \\
(32.77)\end{array}$ & $\begin{array}{c}10.00 \\
(18.41)\end{array}$ & $\begin{array}{c}29.44 \\
(31.86)\end{array}$ & $\begin{array}{c}44.67 \\
(41.92)\end{array}$ & $\begin{array}{c}25.33 \\
(30.21)\end{array}$ & $\begin{array}{c}6.00 \\
(14.14)\end{array}$ & $\begin{array}{r}25.33 \\
(28.76)\end{array}$ \\
\hline$T_{3}$ & $\begin{array}{c}79.33 \\
(62.94)\end{array}$ & $\begin{array}{c}70.67 \\
(57.19)\end{array}$ & $\begin{array}{c}55.00 \\
(47.85)\end{array}$ & $\begin{array}{c}68.33 \\
(55.99)\end{array}$ & $\begin{array}{c}73.33 \\
(58.93)\end{array}$ & $\begin{array}{c}58.33 \\
(49.78)\end{array}$ & $\begin{array}{c}38.67 \\
(38.43)\end{array}$ & $\begin{array}{c}56.78 \\
(49.05)\end{array}$ & $\begin{array}{c}69.33 \\
(56.36)\end{array}$ & $\begin{array}{c}53.33 \\
(46.90)\end{array}$ & $\begin{array}{c}34.67 \\
(36.05)\end{array}$ & $\begin{array}{c}52.44 \\
(46.44)\end{array}$ & $\begin{array}{c}59.00 \\
(50.17)\end{array}$ & $\begin{array}{c}41.67 \\
(40.19)\end{array}$ & $\begin{array}{c}17.00 \\
(24.32)\end{array}$ & $\begin{array}{c}39.22 \\
(38.22)\end{array}$ & $\begin{array}{c}47.33 \\
(43.45)\end{array}$ & $\begin{array}{c}28.67 \\
(32.35)\end{array}$ & $\begin{array}{c}9.00 \\
(17.40)\end{array}$ & $\begin{array}{c}28.33 \\
(31.07)\end{array}$ & $\begin{array}{c}41.67 \\
(40.19)\end{array}$ & $\begin{array}{l}23.00 \\
(28.64)\end{array}$ & $\begin{array}{c}3.67 \\
(11.01)\end{array}$ & $\begin{array}{r}22.78 \\
(26.61)\end{array}$ \\
\hline$T_{4}$ & $\begin{array}{c}79.33 \\
(62.94)\end{array}$ & $\begin{array}{c}70.00 \\
(56.77)\end{array}$ & $\begin{array}{c}54.33 \\
(47.47)\end{array}$ & $\begin{array}{c}67.89 \\
(55.73)\end{array}$ & $\begin{array}{c}73.33 \\
(58.90)\end{array}$ & $\begin{array}{c}58.00 \\
(49.59)\end{array}$ & $\begin{array}{c}37.67 \\
(37.84)\end{array}$ & $\begin{array}{c}56.33 \\
(48.78)\end{array}$ & $\begin{array}{c}69.33 \\
(56.36)\end{array}$ & $\begin{array}{c}53.67 \\
(47.09)\end{array}$ & $\begin{array}{c}33.33 \\
(35.25)\end{array}$ & $\begin{array}{c}52.11 \\
(46.23)\end{array}$ & $\begin{array}{c}58.67 \\
(49.98)\end{array}$ & $\begin{array}{c}41.00 \\
(39.80)\end{array}$ & $\begin{array}{c}16.67 \\
(24.06)\end{array}$ & $\begin{array}{c}38.78 \\
(37.94)\end{array}$ & $\begin{array}{c}45.33 \\
(42.30)\end{array}$ & $\begin{array}{c}27.33 \\
(31.50)\end{array}$ & $\begin{array}{c}8.00 \\
(16.40)\end{array}$ & $\begin{array}{c}26.89 \\
(30.07)\end{array}$ & $\begin{array}{c}41.00 \\
(39.80)\end{array}$ & $\begin{array}{c}22.67 \\
(28.41)\end{array}$ & $\begin{array}{c}3.33 \\
(10.40)\end{array}$ & $\begin{array}{r}22.33 \\
(26.20)\end{array}$ \\
\hline $\mathbf{T}_{5}$ & $\begin{array}{c}80.00 \\
(63.41)\end{array}$ & $\begin{array}{c}72.00 \\
(58.04)\end{array}$ & $\begin{array}{c}57.33 \\
(49.20)\end{array}$ & $\begin{array}{c}69.78 \\
(56.88)\end{array}$ & $\begin{array}{c}73.67 \\
(59.13)\end{array}$ & $\begin{array}{c}59.67 \\
(50.56)\end{array}$ & $\begin{array}{c}40.67 \\
(39.60)\end{array}$ & $\begin{array}{c}\mathbf{5 8 . 0 0} \\
(\mathbf{4 9 . 7 7 )}\end{array}$ & $\begin{array}{c}69.67 \\
(56.57)\end{array}$ & $\begin{array}{c}53.67 \\
(47.08)\end{array}$ & $\begin{array}{c}35.00 \\
(36.25)\end{array}$ & $\begin{array}{c}52.78 \\
(46.64)\end{array}$ & $\begin{array}{c}62.67 \\
(52.32)\end{array}$ & $\begin{array}{c}42.67 \\
(40.77)\end{array}$ & $\begin{array}{c}18.67 \\
(25.58)\end{array}$ & $\begin{array}{l}41.33 \\
(39.55)\end{array}$ & $\begin{array}{l}45.67 \\
(42.50)\end{array}$ & $\begin{array}{c}28.00 \\
(31.93)\end{array}$ & $\begin{array}{c}8.33 \\
(16.77)\end{array}$ & $\begin{array}{c}27.33 \\
(30.40)\end{array}$ & $\begin{array}{c}42.33 \\
(40.57)\end{array}$ & $\begin{array}{c}24.00 \\
(29.31)\end{array}$ & $\begin{array}{c}4.67 \\
(12.35)\end{array}$ & $\begin{array}{r}23.67 \\
(27.41)\end{array}$ \\
\hline \multirow[t]{2}{*}{ Mean } & $\begin{array}{c}79.56 \\
(63.10)\end{array}$ & $\begin{array}{c}70.78 \\
(57.27)\end{array}$ & $\begin{array}{c}55.44 \\
(48.11)\end{array}$ & & $\begin{array}{r}73.56 \\
(59.06)\end{array}$ & $\begin{array}{c}\mathbf{5 8 . 6 1} \\
(\mathbf{4 9 . 9 4 )}\end{array}$ & $\begin{array}{c}38.78 \\
(38.49)\end{array}$ & & $\begin{array}{r}69.56 \\
(56.50)\end{array}$ & $\begin{array}{c}53.44 \\
(46.96)\end{array}$ & $\begin{array}{c}34.94 \\
(36.21)\end{array}$ & & $\begin{array}{c}60.00 \\
(50.77)\end{array}$ & $\begin{array}{c}41.67 \\
(40.18)\end{array}$ & $\begin{array}{c}17.67 \\
\text { (24.80) }\end{array}$ & & $\begin{array}{c}45.89 \\
(42.62)\end{array}$ & $\begin{array}{c}27.89 \\
(31.85)\end{array}$ & $\begin{array}{c}8.50 \\
(16.90)\end{array}$ & & $\begin{array}{c}42.11 \\
(40.44)\end{array}$ & $\begin{array}{r}23.50 \\
(28.97)\end{array}$ & $\begin{array}{c}4.33 \\
(11.86)\end{array}$ & \\
\hline & \multicolumn{4}{|c|}{ C.D. } & \multicolumn{4}{|c|}{ SE(d) } & \multicolumn{4}{|c|}{$\mathbf{S E}(\mathbf{m})$} & \multirow{2}{*}{\multicolumn{4}{|c|}{$\begin{array}{l}\text { C.D. } \\
\text { C.378 }\end{array}$}} & \multicolumn{4}{|c|}{ SE(d) } & \multicolumn{4}{|c|}{$\mathbf{S E}(\mathbf{m})$} \\
\hline $\mathbf{v}$ & \multirow{2}{*}{\multicolumn{4}{|c|}{0.515}} & \multirow{2}{*}{\multicolumn{4}{|c|}{0.260}} & \multirow{2}{*}{\multicolumn{4}{|c|}{0.184}} & \multirow{2}{*}{\multicolumn{4}{|c|}{0.378}} & \multirow{2}{*}{\multicolumn{4}{|c|}{0.191}} & \multirow{2}{*}{\multicolumn{4}{|c|}{0.135}} \\
\hline $\mathrm{L}$ & & & & & & & & & & & & & & & & & & & & & & & & \\
\hline $\mathrm{V \times L}$ & \multirow{2}{*}{\multicolumn{4}{|c|}{$\begin{array}{l}0.892 \\
0.728\end{array}$}} & \multirow{2}{*}{\multicolumn{4}{|c|}{$\begin{array}{l}0.450 \\
0.367\end{array}$}} & \multirow{2}{*}{\multicolumn{4}{|c|}{$\begin{array}{l}0.318 \\
0.260\end{array}$}} & \multirow{2}{*}{\multicolumn{4}{|c|}{$\begin{array}{l}0.654 \\
0.534\end{array}$}} & \multirow{2}{*}{\multicolumn{4}{|c|}{0.33}} & & & & \\
\hline$T$ & & & & & & & & & & & & & & & & & & & & & & & & \\
\hline $\mathrm{VxC}$ & & & & & & 0. & 36 & & & & 45 & & & & & & & 0. & & & & & & \\
\hline LXT & & & 61 & & & 0. & 36 & & & & 45 & & & & & & & 0. & & & & & & \\
\hline $\operatorname{VxLxT}$ & & & & & & 1. & & & & & & & & & & & & & & & & & & \\
\hline & & & & & & & & & & & & $\mathrm{NO}_{3}$ & ${ }_{2} \mathrm{PO}$ & & & & & & & & & & & \\
\hline
\end{tabular}




\section{Seedling establishment (\%)}

Significant differences were observed for the effect of naturally aged Indian mustard seed lot on seedling establishment (Table 3) during both the years of study. Seedling establishment ranged from 38.56-73.89, 28.78-64.00, 16.78-57.89 and 9.11-45.67 per cent during the year 2015-16 and 2016-17, respectively. Maximum seedling establishment (73.89, 64.00, 57.89 and 45.67) was obtained with the seed lot ' $\mathrm{L}_{1}$ ' during both of the years and it was significantly superior over the rest of seed lots, whereas, minimum seedling establishment (38.56, $28.78,16.78$ and 9.11 per cent) was recorded with the seed lot ' $\mathrm{L}_{3}$ ' during the year 2015-16 and 2016-17, respectively. The effect of different seed lots and varieties, found significant results during 2015-16 and 201617 for this attribute. However, maximum seedling establishment (65.67, 55.56, 46.56 and 36.89) was observed in the variety'RH30 ' while minimum seedling establishment (50.67, 39.22, 31.22 and 21.78 per cent) for the variety 'RH-0406' during the year 201516 and 2016-17, respectively. The variety RH-30 had higher seedling establishment followed by RH-9304 and lowest was found in 'RH-0406' after four year of storage under ambient conditions. Seedling establishment for the interaction between varieties and seed lots ranged from 31.33-79.00, 21.33-72.33, $11.00-67.00$ and $4.00-55.33$ per cent during the year 2015-16 and 2016-17. The treatment combination ' $\mathrm{L}_{1} \times \mathrm{RH}-30$ ' observed highest seedling establishment (79.00, 72.33, 67.00 and 55.33 per cent), whereas, lowest seedling establishment $(31.33,21.33,11.00$ and 4.00 per cent) was recorded with the interaction ' $\mathrm{L}_{3}$ ' $\times$ RH-0406' during the year 2015-16 and 2016-17. These results are same in accordance with Rai et al., (2017) in Indian mustard; Singh et al., (2015) in fenugreek; in alfalfa by Cakmak et al., (2010) and in wheat by Chauhan et al., (2011).

\section{Effect of seed priming on field performance of Indian mustard}

\section{Seedling emergence index}

Significant differences were observed for seedling emergence index of naturally aged lots of different varieties of Indian mustard during the year 2015-16 and 2016-17, respectively. Perusal of data (Table 4) indicated that all the treatments were found effective to enhance the seedling emergence index as compared to control in both the year of study. Maximum enhancement in seedling emergence index $(9.84,6.99,6.14$ and 7.03 , $4.23,3.46)$ was recorded with the seed priming treatment ' $\mathrm{T}_{2}$ ' as compared to ' $\mathrm{T}_{0}$ ' in all the seed lots and varieties during the year 2015-16 and 2016-17, respectively. Among the seed lots, ' $\mathrm{L}_{1}$ ' was reported maximum seedling emergence index (10.66, 8.33, 7.69 and $8.64,5.82,5.01)$ followed by $(9.14,6.21$, 4.62 and $6.19,3.56,2.71$ ) in seed lot ' $\mathrm{L}_{2}$ ' and minimum seedling emergence index was recorded $(7.77,4.62,3.64$ and $4.71,1.89$, 1.43) in seed lot ' $\mathrm{L}_{3}$ ' during both the years of study. Among the varieties, maximum enhancement was reported in variety ' $\mathrm{RH}-30$ ' followed by variety 'RH-9304' and minimum enhancement as observed in variety ' $\mathrm{RH}$ 0406' in all the seed lots dung the year 201516 and 2016-17, respectively. Similar findings were observed in fenugreek by Singh et al., (2015) in fenugreek and by Kalsa et al., (2011) in vetch.

\section{Mean emergence time}

Significant differences were observed for mean emergence time of naturally aged seed lots of different varieties of Indian mustard during both the years of study. Perusal of data (4.5) revealed that all the treatment was found superior over the control in all the seed lots and varieties during the year 2015-16 and 2016-17, respectively. Maximum reduction in 
mean emergence time (5.56, 7.72, 8.79 and $6.95,8.97,10.42$ ) was observed with the seed priming treatment ' $\mathrm{T}_{2}$ ' over the priming treatment ' $\mathrm{T}_{0}$ ' (control) in all the seed lots and varieties during both the years of study. Maximum mean emergence time $(6.59,8.92$, 10.17 and $8.33,10.76,11.92$ ) was recorded in seed lot ' $\mathrm{L}_{3}$ ' followed by seed lot ' $\mathrm{L}_{2}$ ' (5.99, 8.08, 9.04 and 7.47, 9.75, 10.89) and minimum mean emergence time $(5.80,7.83$, 8.66 and $6.55,7.81,9.68)$ was recorded in the seed lot ' $\mathrm{L}_{1}$ ' during the year 2015-16 and 2016-17, respectively. Among the varieties, 'RH-30' was performed better with all the seed priming treatment followed by ' $\mathrm{RH}$ 9304' and minimum enhancement was observed in variety ' $\mathrm{RH}-0406$ ' during the year 2015-16 and 2016-17, respectively. These results are same in accordance with Thakre and ghate (1984) in vetch and Verma et al., (2003) in rapeseed and mustard under ambient growing conditions (Table 6).

\section{Seedling establishment (\%)}

Data pertaining to seed priming on seedling establishment of naturally aged seed lots of different of Indian mustard has been presented in Table 6 . All the seed priming treatment was found superior over the control in all the seed lots and varieties during the year 2015-16 and 2016-17, respectively. Among the seed lots, maximum enhancement in seedling establishment $(79.56,73.56,69.56$ and $60.00,45.89,42.11$ per cent) was recorded in seed lot ' $\mathrm{L}_{1}$ ' followed by $(70.78$, $58.61,53.44$ and $41.67,27.89,23.50)$ in seed lot ' $\mathrm{L}_{2}$ ' while, minimum seedling establishment (55.44, 38.78, 34.94 and 17.67, $8.50,4.33$ per cent) was observed in ' $\mathrm{L}_{3}$ ', during both the years of study. The seed priming with treatment ' $\mathrm{T}_{2}$ ' was found effective to enhance the seedling establishment (71.11, 58.89, 54.44 and 42.56, $29.44,25.33$ ) as compared to the control during the year 2015-16 and 2016-17. Among the varieties, maximum enhancement was found in variety ' $\mathrm{RH}-30$ ' followed by variety 'RH-9304' and minimum enhancement was recorded in variety ' $\mathrm{RH}-0406$ ' in all the seed lots dung the year 2015-16 and 2016-17, respectively. Similar findings were observed in fenugreek by Singh et al., (2015) in fenugreek; Kalsa et al., (2011); Thakre and ghate (1984) in vetch and Verma et al., (2003) in rapeseed and mustard under ambient growing conditions.

On the basis of present investigation it is concluded that field performance of Indian mustard studied during both the year was significantly decreased with the advancement of time. Among the different varieties under study, RH-0406 found fast reduction in field emergence and seed establishment, while RH30 was found to be a good storer variety. All the priming treatments enhanced the seed quality significantly in case of field emergence and seedling establishment over the control in naturally aged seed lots of different varieties of Indian mustard. No doubt performance of fresh seed was observed better over all the other lots but, enhancement was comparatively more in marginal seed lot i.e. one year old seed lot $\left(\mathrm{L}_{2}\right)$.Among the seed priming treatments, $\mathrm{GA}_{3}$ was found best performing treatment for improving the seed quality followed by biofertilizer (Azotobecter), hydration and dry dressing with carbendazim $(2 \mathrm{~g} / \mathrm{kg}), \mathrm{KNO}_{3}$ $(0.5 \%)$ and $\mathrm{KH}_{2} \mathrm{PO}_{4}(0.5 \%)$. In overall, the priming technology was found much effective and beneficial for enhancing the field performance and storage potential of Indian mustard seeds.

\section{References}

Anonymous. 2016. Agriculture statistics at a glance, pp 124-125.

Cakmak, T., Atici, O., Agar, G. and Serap, S. 2010. Natural ageing related 
biochemical changes in alfalfa sees stored for 42 years. Int. J. Pl. Sci., 1:16.

Chauhan, D.S., Deswal, D.P., Dahiya, O.S. and Punia, R.C. 2011. Change in storage enzymes activities in natural and accelerated aged seed of wheat (Triticum aestivum). Ind. J. Agric. Sci., 81(11):1037-1040.

Chiu KY, Chuang SJ, Sung JM. 2006. Both anti-oxidation and lipid-carbohydrate conversion enhancements are involved in priming-improved emergence of Echinacea purpurea seeds that differ in size. Sci Hortic. 108(2):220_226.

Ghassemi-Golezani, K., Bakhshy, J., Raey, R. and Hossainzadeh-Mahootchy, A. 2010. Seed vigour and field performance of winter oilseed rape (Brassica napus L.) cultivars. Not. Bot. Hort. Agrobot. Cluj., 38(3):146-150.

Gomez, K.A. and Gomez, A.A. (1984). Statistical. Procedure. for Agricultural. Research., $2^{\text {nd }}$ ed. John Wiley and Sons, New York, USA.

Hsu CC, Chen CL, Chen JJ, Sung JM. 2003. Accelerated aging enhanced lipid peroxidation in bitter gourd seeds and effects of priming and hot water soaking treatments. Sci Hortic. 98(3): 201-212.

Kalsa, K.K., Tomer, R.P.S. and Abebie, B. 2011. Effects of storage duration and hydro-priming on seed germination and vigour of common vetch. J. Sci. \& Develop., 1(1):65-73.

Khajeh-Hosseini M, Powell AA, Bingham I.J. 2003. The interaction between salinity stress and seed vigor during germination of soybean seeds. Seed Sci Technol. 31(3):715_725.

Khajeh-Hosseini, M., Nasehzadeh, M. and Matthews, S. 2010. Rate of physiological germination relates to the percentage of normal seedlings in standard germination tests of naturally aged seed lots of oilseed rape. Seed
Science and Technology, 38(3): 602611.

Lanteri S, Quagliotti L, Belletti P. 1998. Delayed luminescence and priminginduced nuclear replication of unaged and controlled deteriorated pepper seeds (Capsicum annuum L.). Seed Sci Technol. 26(2):413_ 424.

McDonald MB. 2000. Seed technology and its biological basis. Sheffield (AC): Sheffield Academic Press. Chapter 5, Seed priming; p. 287_325.

Panse, V.G. and Sukhatme, P.V. 1961. Statistical Methods for Agricultural Workers. Indian Council of Agricultural Research, New Delhi, India, 381 p.

Patane C, Cavallaro V, Cosentino SL. 2009. Germination and radicle growth in unprimed and primed seeds of sweet sorghum as affected by reduced water potential in $\mathrm{NaCl}$ at different temperatures. Ind Crops Prod. 30(1):18.

Rai Himanshu., Peerzada Ovais Hamid, Dahiya, O.S. and Jakhar, S.S. 2017. Seed vigour assessment in different varieties of Indian mustard. Inj. J. Curr. Microbio. App. Sci. 6(10): 1930-1936.

Sadeghian SY, Yavari N. 2004. Effect of water-deficit stress on germination and early seedling growth in sugar beet. $J$ Agron Crop Sci. 190(2):138-144.

Singh Bahader, Bhuker Axay, Mor, V.S., Dahiya, O.S. and Punia, R.C. 2015. Effect of Natural Ageing on Seed Quality of Fenugreek (Trigonella foenumgraecum L.). International Journal of Scientific Research in Science and Technology, (1)4:243-248.

Soltani, E., Galeshi, S., Kamkar, B. and Akramghaderi, F. 2009. The Effect of Seed Aging on the Seedling Growth as Affected by Environmental Factors in wheat. Res. J. Environ. Sci., 3:184- 192.

Soltani, E., Kamkar, B., Galeshi, S., Akram and Ghaderi, F. 2008. The effect of seed 
deterioration onseed reserves depletion $\mathrm{n}$ and heterotrophic seedling growth of wheat. J. Agric. Sic. Natur. Resour., 15(1):13-17.

Thakre, S.K. and Ghate, N.N. 1984. Effect of seed soaking with hormones on grain and fodder yield of sorghum and uptake of nutrients by the crop. PKU Res. J., 8:10-12.

Verma, S.S., Verma, U. and Tomer, R.P.S. 2003. Studies on seed quality parameters in deteriorating seeds in brassica (Brassica campestris). Seed Sci. \& Technol., 31:389-396.

\section{How to cite this article:}

Pradeep Singh, R.C. Punia, V.S. Mor and Sunil Kumar. 2018. Effect of Natural Ageing and Seed Priming on Field Emergence of Indian Mustard [Brassica juncea L. (Czern \& coss)]. Int.J.Curr.Microbiol.App.Sci. 7(03):3227-3236.

doi: https://doi.org/10.20546/ijcmas.2018.703.373 